\title{
AZIMUTHAL RESISTIVITY STUDIES USING THE OFFSET WENNER SOUNDING TECHNIQUE
}

\author{
K. A. Watson \& R. D. Barker \\ School of Earth Sciences, University of Birmingham, Edgbaston, Birmingham, B15 2TT, UK. \\ Email: watson@ers.bham.ac.uk
}

\section{INTRODUCTION}

In recent years, hydrogeologists have employed electrical surveying principles to detect and characterise fracture induced anisotropy. In particular, azimuthal resistivity surveying has been adopted (Leonard-Mayer (1984), Taylor and Fleming (1988), Ritzi and Andolsek (1992), Skjernaa and Jergensen (1993), al Hagrey (1994)) as a technique for determining the principal directions of electrical anisotropy and hence hydraulic conductivity. However, in most recent published examples of the application of azimuthal resistivity surveys, the technique has been poorly applied with results almost certainly wrongly interpreted. In this short paper we emphasise some of the pitfalls and suggest methods of eliminating serious sources of interpretational ambiguity.

\section{AMBIGUITIES IN THE INTERPRETATION OF AZIMUTHAL DATA}

Azimuthal resistivity surveys have been widely applied in recent years with any observed change in apparent resistivity with azimuth being interpreted as indicative of anisotropy (generally fracture anisotropy). However azimuthal variations in apparent resistivity may also originate from the presence of a dipping interface or a gradational lateral change in resistivity or indeed any combination of these. Most published examples of azimuthal surveying have overlooked this and consequently it is likely that the observed response has been misinterpreted on many occasions.

Anisotropy can be considered to possess a 180 degree symmetry, in that its properties are invariant when it is rotated through this angle; on the other hand, dip and lateral effects possess a 360 degree symmetry. In order to successfully differentiate between these two types of symmetry, an azimuthal technique must be employed which also has a 360 degree symmetry. One such appropriate method is the Offset Wenner technique (Barker, 1981).

\section{THE AZIMUTHAL OFFSET WENNER TECHNIQUE}

The Offset Wenner system of resistance measurement uses a collinear arrangement of five equally spaced electrodes, with resistances being measured first with the left four electrodes $\left(R_{D 1}\right)$ and then with the right four electrodes $\left(\mathrm{R}_{\mathrm{D}_{2}}\right)$. Analysis of the relative behaviour of these two Wenner resistances as a function of both azimuth and electrode spacing, enables differentiation between true anisotropy and other geological models (Nunn et al., 1983).

Consider four simple geological models; (1) a homogeneous isotropic earth, (2) a homogeneous anisotropic earth, (3) a dipping interface separating two homogeneous isotropic layers and (4) a laterally changing resistivity model.

Over a horizontally layered subsurface $R_{D 1}$ and $R_{D 2}$ are equal at all azimuths and depths, whether or not the ground is anisotropic. In stark contrast, vertical (or sub-vertical) anisotropy can be identified as the two resistances rise and fall simultaneously as a function of azimuth. In this instance, the greatest resistance observed occurs parallel to the fracture direction, in accordance with paradox 
of anisotropy. As in the previous case, the concurrent relationship between $R_{D 1}$ and $R_{D 2}$ is independent of depth. Provided the anisotropy persists at depth the overall magnitude, or range, observed in $R_{D 1}$ and $R_{D 2}$ will also remain unchanged.

Above a dipping interface (separating two homogeneous and isotropic units of different resistivities the values of $R_{D 1}$ and $R_{D 2}$ will differ significantly according to the orientation of the offset configuration. When parallel to the strike of the interface, $R_{D 1}$ and $R_{D 2}$, are equal; this point is represented by a cross-over point on the azimuth plot. Maximum variation between the two resistances is observed 90 degrees from this, when the array is aligned in the direction of true dip. However, this relationship is intrinsically related to the depth of investigation. For example, at small electrode spacings, the influence of the dipping interface is negligible and $R_{D 1}$ will equal $R_{D 2}$, as in the isotropic case. As the electrode spacings increase however, the presence of the interface impacts on the measured response and the two resistances become offset from one another in the manner described. At very large electrode spacings the depth of investigation exceeds that of the interface, and $R_{D 1}$ and $R_{D 2}$ can be observed to resume the isotropic relationship.

When Offset Wenner azimuthal soundings are conducted above coarsening sedimentary deposits, or indeed any geological model which exhibits a continuous, uniform lateral change in resistivity, $R_{D 1}$ and $R_{D 2}$ exhibit a similar relationship to that observed above a dipping interface. Again, the greatest difference between $R_{D 1}$ and $R_{D 2}$ occurs in the direction of greatest lateral change and perpendicular to this they are equal. The range between the two resistances also increases as a function of depth but, in this simple case, does not attain the maximum seen in the instance of a single dipping interface.

\section{FIELD STUDIES}

Azimuthal resistivity surveys have been carried out at several sites in the UK and abroad. These have typically consisted of Offset Wenner depth soundings conducted at 20 degree intervals (usually from 000 to 180 ) about a common centrepoint. A complete suite of resistance measurements were made along each azimuth (from $1 \mathrm{~m}$ to $48 \mathrm{~m}$ or greater electrode spacings) using a digital earth resistance meter and Campus Offset Wenner multicore cables. At each site, the relative positions of $R_{D 1}$ and $R_{D 2}$ were noted.

\section{FIELD EXAMPLE OF AN ANISOTROPIC RESPONSE}

This survey was conducted in an area of almost vertically bedded (dip 85 degrees) Carboniferous Limestone beds striking E-W. Figure 1 contains azimuthal plots of the percentage resistance (for electrode spacings of 4,6 and $8 \mathrm{~m}$ ) in both cartesian and polar co-ordinates. Polar plots of $R_{D}$, the mean of $R_{D 1}$ and $R_{D 2}$, are also presented.

In the azimuth plots corresponding to 4 and $6 \mathrm{~m}, \mathrm{R}_{\mathrm{D} 1}$ and $\mathrm{R}_{\mathrm{D} 2}$ are clearly seen to rise and fall together as a function of azimuth. The maximum resistance observed occurs at approximately $100 / 280$ (averaging $R_{D 1}$ and $R_{D 2}$ ); successfully delineating the strike of the beds. The anisotropy recorded at these spacings is quite significant, with anisotropy coefficients $(\lambda)$ of 1.38 and 1.67 respectively.

At an electrode spacing of $8 \mathrm{~m}$ the relationship between $R_{D 1}$ and $R_{D 2}$ alters slightly, as whilst the two graphs retain the position and magnitude of their peak resistance, they have become offset from one another. Azimuthal plots at greater electrode spacings proceed to adopt a form representative of a dipping interface, the strike of which occurs at an orientation of 100/280. This result is particularly satisfyingly as it can be justified geologically. The two units present at this location possess a conformable contact, coplanar with the bedding in the overlying limestone. This explains why the interface strike occurs at the same azimuth as the peak resistances (bedding strike) in the 4 and $6 \mathrm{~m}$ azimuths plots. 


\section{FIELD EXAMPLE OF A DIPPING INTERFACE RESPONSE}

This survey was performed in County Galway, Eire, in an area of Carboniferous Limestone. This survey site was selected as it had been anticipated that the azimuthal technique would detect the anisotropy associated with the solution enlarged joints and bedding planes of the formation. Fig. 2 contains the observed responses at electrode spacings of $12 \mathrm{~m}, 16 \mathrm{~m}$ and $24 \mathrm{~m}$.

Consider, initially, the response of the traditional four-electrode Wenner configuration (column (c)), obtained from the mean of $R_{D 1}$ and $R_{D 2}$. The polar plots of $R_{D}$ in this instance clearly suggest a directional preference in the resistivity distribution. Given the geological terrain, it is not unreasonable to conclude that anisotropic ground (where $\lambda=1.07$ ) has been encountered. However, if $R_{D 1}$ and $R_{D 2}$ are considered separately, the azimuthal pattern of potential alters considerably, and the characteristic response of a dipping interface becomes evident. The cartesian form of data display generally lends itself best to visual interpretation. From this it is apparent that the cross-over points consistently identify the strike of the dipping interface as 090/270 and that maximum variation in the two resistances occurs at an orientation of 160/340. Furthermore, the interface can be shown to dip southwards, as at this azimuth it had been noted that $R_{D 1}$ was positioned north of $R_{D 2}$. Analysing the complete data set in terms of the magnitude of the range in $R_{D 1}$ and $R_{D 2}$ further confirms the presence of a dipping interface.

Confirmation of the subsurface structure was achieved through electrical resistivity imaging of the site. The southerly dip of the limestone surface is clearly evident in the resultant model resistivity pseudosection.

\section{CONCLUSIONS}

In this paper, it has been illustrated that apparently anisotropic distributions of apparent resistivity can be generated by a variety of quite different geological scenarios and that consequently the detection of fracture anisotropy is not as straightforward as previously accepted.

The response of the Offset Wenner azimuthal technique above the simple geological models presented in this paper, illustrates that this alternative approach has the ability to remove such interpretational ambiguities and can discriminate between true anisotropy and pseudo-anisotropic lateral influences. Field data collected with this technique have further demonstrated its practical application.

\section{REFERENCES}

Barker, R. D. 1981. The offset system of electrical resistivity sounding and its use with a multicore cable. Geophysical Prospecting, 29, 128 - 143.

Hagrey, S. A. 1994. Electric study of fracture anisotropy at Falkenberg, Germany. Geophysics, 59, No. 6, $881-888$.

Leonard-Mayer, P. 1984. A surface resistivity method for measuring hydrologic characteristics of jointed formations. Report of Investigations 8901, US Department of the Interior.

Ritzi, R. W. and Andolsek, R. H. 1992. Relation between anisotropic transmissivity and azimuthal resistivity surveys in shallow fractured, carbonate flow systems. Ground Water, 30, No. 5, $774-780$.

Skjernaa, L. and Jorgensen, N. O. 1993. Detection of local fracture systems by azimuthal resistivity surveys: examples from south Norway. Memoires of the 24th Congress of IAH, Oslo.

Taylor, R. W. and Fleming, A. H. 1988. Characterising jointed systems by azimuthal resistivity surveys. Ground Water, 26, No. 4, 464 - 474.

Nunn, K. R., Barker, R. D. and Bamford, D. 1983. In-situ seismic and electrical measurements of fracture anisotropy in the Lincolnshire chalk. Quarterly Journal of Engineering Geology, $16,187-195$ 


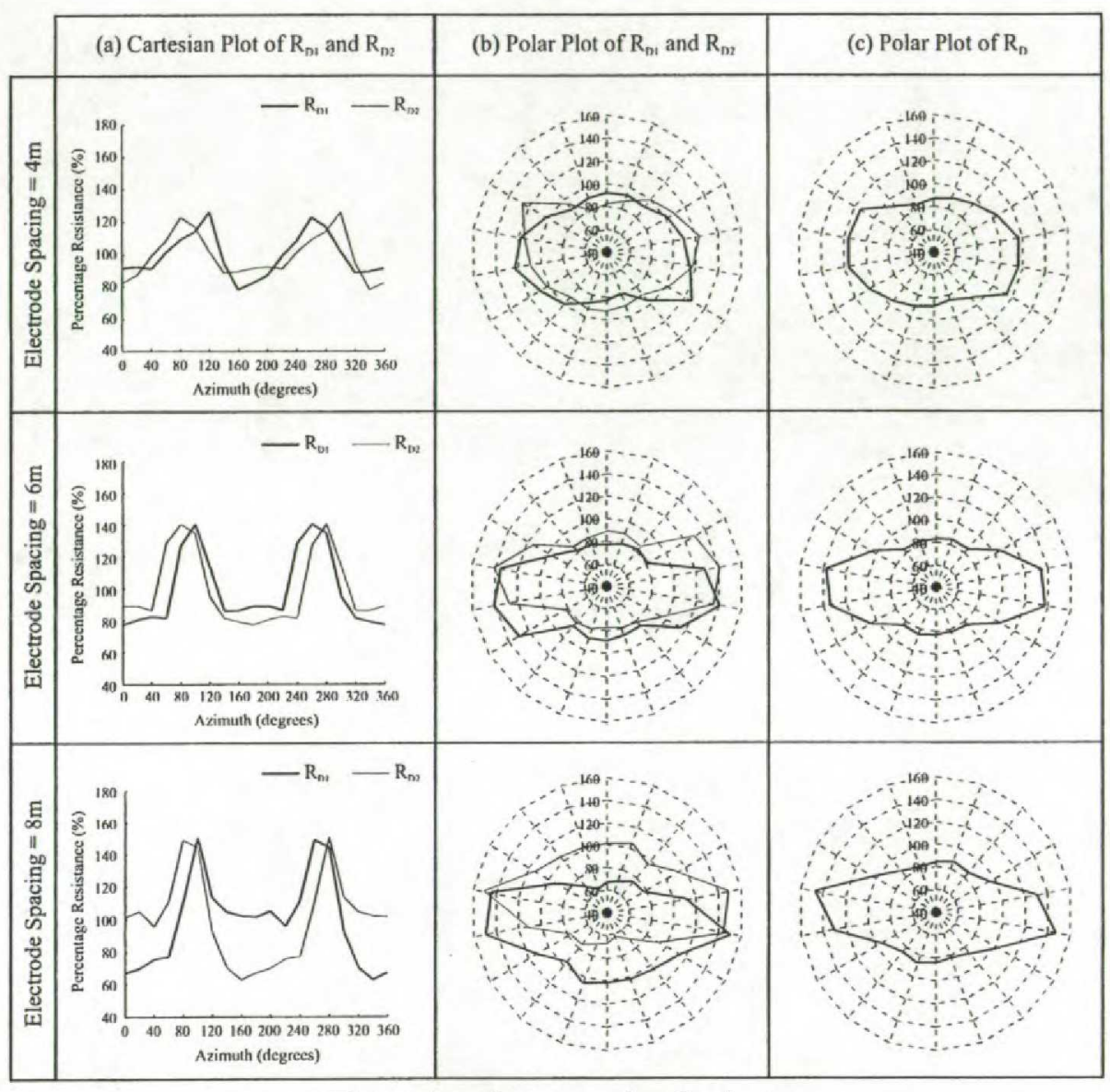

Figure 1. An example of anisotropy

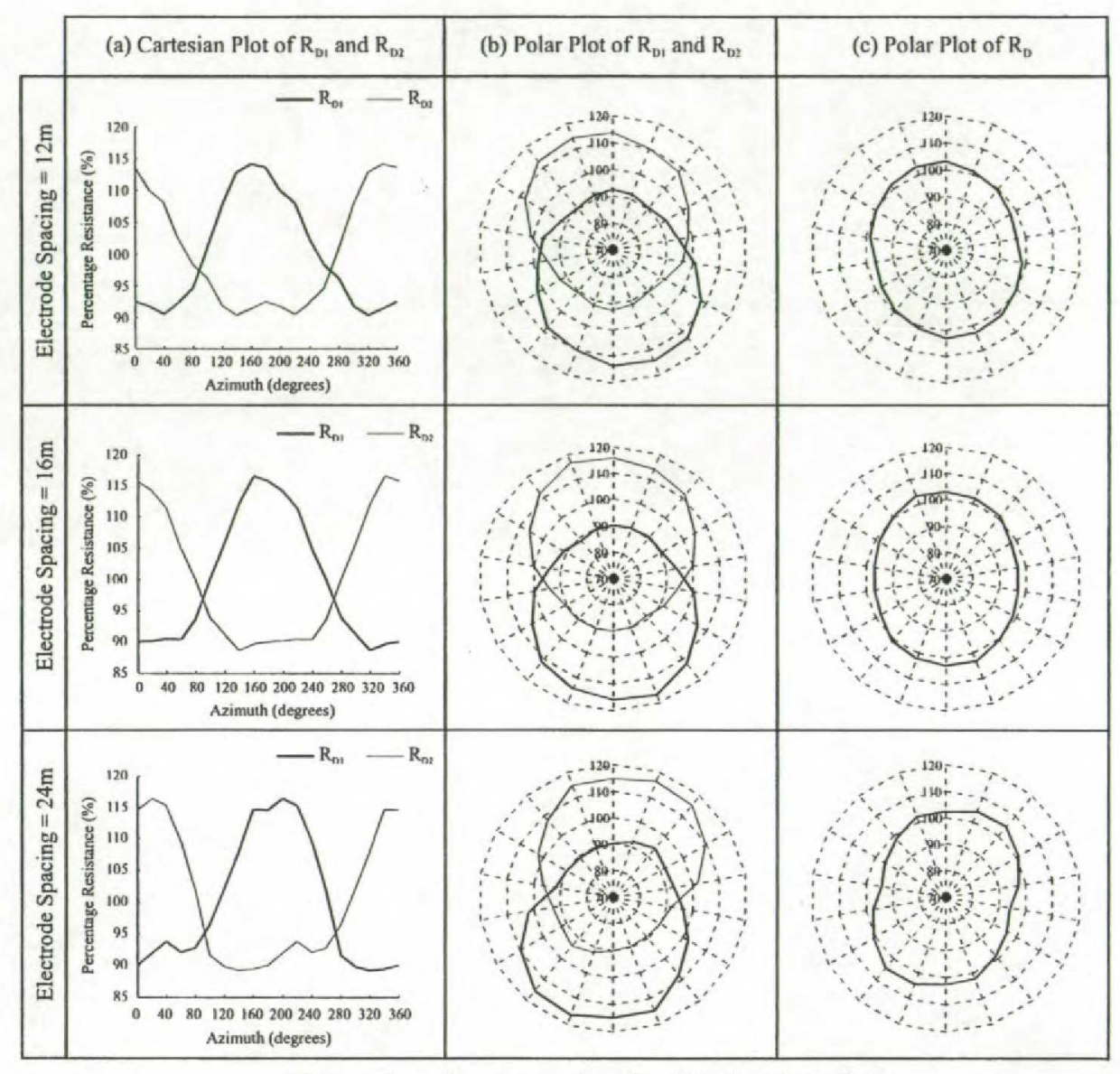

Figure 2. An example of a dipping interface 